\title{
PART 12.
}

\section{Outflows From YSO And AGN}


Accretion Phenomena and Related Outflows, IAU Colloquium 163

ASP Conference Series, Vol. 121, 1997

D.T. Wickramasinghe, L. Ferrario, and G.V. Bicknell, eds.

\title{
Magnetized Accretion Disks and the Origin of Bipolar Outflows
}

\author{
Arieh Königl \\ Department of Astronomy \& Astrophysics, University of Chicago, 5640 \\ S. Ellis Ave., Chicago, IL 60637, U.S.A.
}

\begin{abstract}
Centrifugally driven winds from the surfaces of magnetized accretion disks are an attractive mechanism for removing the angular momentum of the accreted matter and of producing bipolar outflows and jets in compact astronomical objects. In this contribution, I first review steady-state disk-wind models that have been constructed for the different density regimes of circumstellar disks and comment on their expected stability. I then consider several nonsteady effects, including disk formation in molecular cloud-core collapse, magnetic flux transport through the disk, and the role of magnetic fields in the FU Orionis outburst phenomenon. I conclude with a discussion of some of the unique observational properties of disk-driven outflows in young stellar objects and in active galactic nuclei. These characteristics are a consequence of the highly stratified density and velocity structures of centrifugally driven outflows, their large momentum discharges (which result in the efficient uplifting of dust from the disk), and, in the case of molecular disks around lowluminosity objects, their comparatively low initial degrees of ionization (which can lead to rapid heating by ambipolar diffusion).
\end{abstract}

\section{Introduction}

Centrifugally driven winds (CDWs) from the surfaces of magnetized accretion disks have been recognized as a potentially efficient means of tapping the rotational kinetic energy of the disk material and of carrying away its angular momentum. These properties, in turn, have made them a leading candidate for the origin of the energetic bipolar outflows and jets that are observed in young stellar objects (YSOs), active galactic nuclei (AGNs), as well as in other compact astronomical systems (e.g., Königl \& Ruden 1993; Blandford 1993). The ability of such outflows to exert a strong braking torque on the disk may have important implications to the fundamental question of the angular momentum transport mechanism in astrophysical accretion disks. In fact, it has been suggested that, in this respect, CDWs are crucial for the accretion process and that this may account for the ubiquity of bipolar outflows and for the observationally inferred strong link between disks and jets (e.g., Königl 1994).

The basic outflow mechanism from a disk that is threaded by open magnetic field lines can be understood with the help of a mechanical analogy to a bead on a rotating, rigid wire. As Blandford \& Payne (1982) showed in the case of a Keplerian rotation law, the bead (representing the matter) will be flung out if 
the wire (representing the magnetic field line in the ideal MHD limit) is inclined at an angle $<60^{\circ}$ to the disk plane. Using a self-similar (in the spherical radial coordinate), cold wind model, they constructed explicit solutions of flows that are magnetically dominated above the disk surface and become super-Alfvénic and magnetically collimated further out. The angular momentum transport efficiency of the wind can be traced to the fact that any given field line (rotating with the angular velocity of the material at the radius $r_{0}$ where it leaves the disk) enforces corotation out to the (cylindrical) Alfvén radius $r_{A}$, thereby increasing the lever arm of the torque that acts back on the disk. Since $r_{\mathrm{A}}$ could be $\gg r_{0}$, the mass outflow rate that is required for the removal of all the liberated angular momentum might be only a small fraction of the mass accretion rate: $\dot{M}_{\text {wind }} / \dot{M}_{\text {acc }} \approx\left(r_{0} / r_{\mathrm{A}}\right)^{2}$. The terminal (poloidal) speed of the wind is typically $\sim\left(r_{0} / r_{\mathrm{A}}\right)$ times the Keplerian speed $V_{\mathrm{K} 0}$ at $r_{0}$, and hence the kinetic power carried by the outflow from a narrow slice of the disk that is centered at $r_{0}$ is $\sim 0.5 \dot{M}_{\mathrm{acc}} V_{\mathrm{K} 0}^{2}$. The wind may thus also transport a large fraction of the gravitational potential energy liberated in the disk.

Various authors have explored more general CDW configurations, both selfsimilar and non-self-similar (see, e.g., Königl 1995 for a review), but the essential features of these solutions are nevertheless captured by the basic Blandford \& Payne model. In this picture, the toroidal magnetic field component is not important in driving the outflow near the disk surface, although it plays a central role in collimating the wind by hoop stresses beyond the Alfven point. It is in principle possible for magnetized outflows to be driven from the start by the gradient in $B_{\phi}$ (e.g., Lovelace et al. 1991; Contopoulos 1995), but such winds might be hard to maintain in a steady state (e.g., Cao \& Spruit 1994) and will not be further considered here.

\section{Magnetized Disk Models}

Several attempts have been made in recent years to construct self-consistent models of magnetized, wind-driving accretion disks and to explore their properties. These efforts have focused on rotationally supported disks that are threaded by large-scale, open magnetic field lines and in which the angular momentum transport is dominated by centrifugally driven outflows from the disk surfaces. In a steady state, these models must incorporate magnetic diffusivity to counter the radial advection and azimuthal shearing of the field. Such models have been developed for weakly ionized disks (e.g., Königl 1989; Wardle \& Königl 1993; $\mathrm{Li}$ 1996) as well as for ionized, resistive disks (e.g., Königl 1994; Ferreira \& Pelletier 1995; Li 1995), and are discussed below in the cotext of a protostellar environment. The stability properties of these disk configurations have not yet been properly explored, although they appear to be immune to the most disruptive types of instability (e.g., Königl \& Wardle 1996). In particular, the magnetic field in these models, whose magnitude is essentially determined from the condition that all the angular momentum liberated by the accreting matter is transported by a CDW, is found to automatically lie in a 'stability window', in which it is strong enough not be affected by the magnetic shearing instability but not so strong as to be subject to the radial interchange instability. 


\subsection{Weakly Ionized Disks in YSOs}

A rotating disk that is threaded by a vertical magnetic field can be regarded as a giant battery, with the potential drop across the disk $\left(\propto \int_{r_{\text {in }}}^{r_{\text {out }}} V_{\phi} B_{z} d r\right)$ driving a radial electric current that flows in an electric circuit that closes outside the disk. For typical protostellar disk parameters around solar-mass YSOs, one can distinguish between the low-density regime (on scales $r \lesssim 100 \mathrm{AU}$ ), in which the current in the disk is carried by metal ions and electrons that recombine on grain surfaces, and the high-density regime (on scales $r \lesssim 1 \mathrm{AU}$ ), where the current is carried by small charged grains or by ions and electrons that recombine without grains (with the latter possibility applying in case the grains have already settled down to the disk midplane). Table 1 gives a schematic representation of such disks and lists the relevant diffusion mechanisms that operate in the various regimes. As discussed in Wardle's contribution to these Proceedings, the accretion on scales $\leqslant 10 \mathrm{AU}$ is expected to occur only in 'active' surface layers, with the inert interior being too weakly ionized for the field to be effectively coupled to the matter.

Table 1. Representative YSO Disk Parameters

\begin{tabular}{ccccc}
\hline $\log (r / \mathrm{AU}):$ & -1 & 0 & 1 & 2 \\
$T\left({ }^{\circ} \mathrm{K}\right):$ & $10^{4 \S}$ & 300 & 100 & 30 \\
$\log \left(n_{0} / \mathrm{cm}^{-3}\right):^{\dagger}$ & 17 & $14.5\left(13^{\ddagger}\right)$ & 12 & 10 \\
$\log \left(B_{0} / G\right):^{\dagger}$ & 1 & 0 & -1 & -2 \\
$\log \left(B_{\mathrm{eq}} / G\right):^{\sharp}$ & $3^{\S}$ & $1\left(0.5^{\ddagger}\right)$ & -0.2 & -1 \\
Composition: & $i-e^{\S}$ & $n-g_{+}-g_{-}$ & $n-i-g_{-}$ & $n-i-e-g_{-}$ \\
& & $($or $n-i-e)$ & & \\
Diffusion & anomalous & ambipolar diff'n & ambipolar & diffusion \\
mechanism: & Ohmic ${ }^{\S}$ & (in 'active' layers) & (plus $g_{-}-n \&$ & $e-i$ drifts) \\
\hline
\end{tabular}

\footnotetext{
'During the 'high' phase of thermal ionization instability.

${ }^{\dagger}$ The symbols $n_{0}, B_{0}, n, i, e, g_{+}$, and $g_{-}$denote, respectively, the midplane density, midplane magnetic field, neutral particles, metal ions, electrons, positively \& negatively charged grains. ${ }^{\ddagger}$ At base of 'active' accretion layers.

"Equipartition field defined by $B_{\text {eq }} \equiv\left(8 \pi n_{0} k T\right)^{0.5}$.
}

Consider a region in a YSO disk where the main charge carriers are metal ions and electrons and where the dominant particles are neutrals (denoted by the subscripts $\mathrm{i}$, e, and $\mathrm{n}$, respectively), and ignore, for simplicity, any dynamical effect of grains. The magnetic field is 'frozen' into the electrons and diffuses relative to the neutrals as a result of either an ion-neutral drift (ambipolar 
diffusion) or an electron-ion $\left(\mathbf{E}^{\prime} \times \mathbf{B}\right)$ drift (the Hall current). The flux freezing condition breaks down when Ohmic dissipation (associated mainly with $e-$ $n$ collisions) becomes important. These three processes enter the generalized Ohm's law in the form

$$
\mathbf{E}^{\prime} \equiv \mathbf{E}+\frac{\mathbf{V} \times \mathbf{B}}{c}=\frac{\mathbf{j}+\beta_{\mathrm{e}} \mathbf{j} \times \mathbf{b}+\beta_{\mathrm{e}} \beta_{\mathrm{i}}\left(\rho_{\mathrm{n}} / \rho\right)^{2} \mathbf{b} \times(\mathbf{j} \times \mathbf{b})}{\sigma} .
$$

In this expression, $\mathbf{j}$ is the current density, $\mathbf{E}^{\prime}$ is the electric field in a frame moving with the bulk velocity $\mathbf{V}$ (effectively the neutral velocity $\mathbf{V}_{n}$ ), $\beta_{\mathrm{e}} \equiv$ $\omega_{\mathrm{ce}} / \nu_{\mathrm{en}}$ (where $\omega_{\mathrm{ce}}=e B / m_{\mathrm{e}} c$ is the electron cyclotron frequency and $\nu_{\mathrm{en}} \propto$ $n$ is the electron-neutral collision frequency) and $\beta_{\mathrm{i}} \equiv \omega_{\mathrm{ci}} / \nu_{\text {in }} \equiv q \beta_{\mathrm{e}}$ (where $q \approx 1.0 \times 10^{-4} T^{0.5}, T$ being the temperature) are the electron and ion Hall parameters, $\sigma \approx \sigma_{\mathrm{e}}=e^{2} n_{\mathrm{e}} / m_{\mathrm{e}} \nu_{\mathrm{en}}$ is the electrical conductivity, and $\mathbf{b} \equiv \mathbf{B} / B$. The Hall parameters determine how well the ionized components are coupled to the magnetic field, with $\beta>1$ signifying good coupling. The three terms on the right-hand side of this equation represent the effects of Ohmic diffusivity, the Hall current, and ambipolar diffusion, respectively. By comparing these terms, one can identify the following regimes:

$$
\begin{array}{rc}
\beta_{\mathrm{i}}>1: & \text { pure ambipolar diffusion } \\
\sqrt{q}<\beta_{\mathrm{i}}<1: & \text { Hall-modified ambipolar diffusion } \\
q<\beta_{\mathrm{i}}<\sqrt{q}: & \text { Hall-modified Ohmic diffusivity } \\
\beta_{\mathrm{i}}<q\left(\leftrightarrow \beta_{\mathrm{e}}<1\right): & \text { pure Ohmic diffusivity. }
\end{array}
$$

In delineating the parameter ranges that lead to self-consistent disk-wind solutions, one needs to consider also the neutral-ion coupling parameter, given by $\eta \equiv \gamma \rho_{\mathrm{i}} r / V_{\mathrm{K}}$, the ratio of the dynamical time to the neutral-ion coupling time $1 / \gamma \rho_{\mathrm{i}}$ (where $\gamma \approx 3.5 \times 10^{13} \mathrm{~cm}^{3} \mathrm{~g}^{-1} \mathrm{~s}^{-1}$ is the collisional coupling coefficient; $\eta>1$ indicates good coupling). One can carry out the analysis by utilizing the hydrostatic approximation first employed by Wardle \& Königl (1993) and further extended by Königl (1994). In this approach, the problem is simplified by neglecting the vertical component of the neutral velocity (a good approximation throughout much of the disk column), which transforms the radial and azimuthal components of the neutral momentum equation into algebraic relations. In the pure ambipolar diffusion regime, this procedure yields the following parameter constraints on viable solutions:

$$
(2 \eta)^{-1 / 2} \lesssim a \lesssim 2 \lesssim \epsilon \eta \lesssim V_{\mathrm{K}} / 2 C_{\mathrm{s}} .
$$

Here $a \equiv B_{0} /\left(4 \pi \rho_{0}\right)^{1 / 2} C_{\mathrm{s}}$ is the ratio of the midplane (subscript 0$)$ Alfvén speed to the isothermal sound speed $C_{\mathrm{s}}$, and $\epsilon \equiv-V_{\mathrm{r} 0} / C_{\mathrm{s}}$ is the normalized midplane inflow speed. The first inequality corresponds to the requirement that the disk remain sub-Keplerian, the second to the wind-launching condition at the disk surface (subscript $b$ ) $B_{r}\left(Z_{\mathrm{b}}\right) \gtrsim B_{0} / \sqrt{3}$ (see $\S 1$ ), and the third to the requirement that the base of the wind lie well above a density scale height in the disk. ${ }^{1}$ The

\footnotetext{
${ }^{1}$ The second and third inequalities together imply that the vertical magnetic stress dominates the gravitational tidal stress in confining the disk: this is a generic property of this class of disk solutions that does not depend on the nature of the magnetic diffusivity.
} 
last inequality expresses the requirement that the ambipolar diffusion heating rate at the midplane not exceed the rate $\rho_{0}\left|V_{r 0}\right| V_{\mathrm{k}} / 2 r$ of gravitational potential energy release. In turn, $C_{\mathbf{s}} / V_{\mathrm{K}}$ must be $\ll 1 /(1+\epsilon)$ to guarantee that the disk is in near-Keplerian motion and geometrically thin. One can also place upper limits on the density at the sonic point to insure that the bulk of the disk material is hydrostatic and that $\dot{M}_{\text {wind }}$ does not exceed $\dot{M}_{\text {acc. }}$. Similar constraints are derived when this analysis is applied to the other diffusivity regimes identified above. It turns out that there are 4 distinct cases in the Hall-modified regime, defined by the products $\left|2 \eta \beta_{\mathrm{i}}\right|$ and $\beta_{\mathrm{i}} \beta_{\mathrm{e}}$ being $>1$ or $<1$, respectively. In the purely Ohmic regime, this analysis implies 3 viable cases: $\eta>\left(2 \beta_{\mathrm{i}} \beta_{\mathrm{e}}\right)^{-1}$, $\left|2 \beta_{\mathrm{i}}\right|^{-1}<\eta<\left(2 \beta_{\mathrm{i}} \beta_{\mathrm{e}}\right)^{-1}$, and $\left(3 \beta_{\mathrm{e}}^{2}\right)^{-1}<\eta<\left|2 \beta_{\mathrm{i}}\right|^{-1}$. Interestingly, the analytic approximations indicate that, in some of these parameter regimes, the simple wind-launching condition $B_{r}\left(Z_{\mathrm{b}}\right) \gtrsim B_{0} / \sqrt{3}$ must be modified, although the original constraint is recovered if $\eta \beta_{\mathrm{i}} \propto n_{\mathrm{i}} / n$ becomes sufficiently large near the top of the disk. Analogous results are obtained in the grain-dominated region of the disk (where, for equal positively and negatively charged grain masses, the Hall term in Ohm's law is identically zero).

\subsection{Ionized Resistive Disks}

Magnetic diffusivity in the disk is accompanied by Joule dissipation, which heats the gas at a rate

$$
\Gamma_{\text {Joule }}=\mathbf{j} \cdot \mathbf{E}^{\prime}=j^{2} / \sigma+\beta_{\mathrm{e}} \beta_{\mathrm{i}}\left(\rho_{\mathrm{n}} / \rho\right)^{2}|\mathbf{j} \times \mathbf{b}|^{2} / \sigma .
$$

It is conceivable that this heating could maintain the requisite degree of ionization for efficient gas-field coupling in the innermost regions of the disk (e.g., Li 1996). In particular, when the temperature rises to $\sim 10^{3} \mathrm{~K}$, potassium can be efficiently ionized. A preliminary exploration of the parameter space of viable disk-wind solutions has indicated that this process might be important on scales of $\sim 0.1 \mathrm{AU}$. However, it appears that on smaller scales the degree of ionization attained in this manner is too low to keep the coupling parameter $\eta$ above 1 , implying that self-consistent disk-wind configurations cannot be sustained in the absence of another ionization mechanism.

A possible mechanism for recoupling the gas to the field in the vicinity of the YSO is the thermal ionization instability originally discussed in the context of dwarf novae and more recently invoked as a possible explanation of FU Orionis outbursts (e.g., Bell \& Lin 1994). In this picture, accretion in the innermost disk proceeds in a nonsteady fashion, with a 'gate' at $r \lesssim 0.25 \mathrm{AU}$ opening every $\sim 10^{3} \mathrm{yr}$ or so after the accumulated column density has become large enough to trigger the instability. During the 'high' phase of the instability (which is identified with an outburst) the gas is hot $\left(T \gtrsim 10^{4} \mathrm{~K}\right)$ and almost completely ionized, and mass rains in at a rate $\gtrsim 10^{-5} M_{\odot} \mathrm{yr}^{-1}$, whereas during the 'low' phase the temperature and degree of ionization decline sharply, and the accretion rate drops to $\lesssim 10^{-7} M_{\odot} \mathrm{yr}^{-1}$. In the context of the magnetized disk model, one can attribute the increase in the accretion rate as the gas becomes highly ionized to the reestablishment of good coupling between the field and the matter, which allows the field to extract the angular momentum of the accreting gas. This could account both for the marked increase in $\dot{M}_{\text {in }}$ and for the strong disk outflow that evidently accompanies it (e.g., Calvet et al. 1993). 
As was pointed out above, it is possible to construct self-consistent diskwind solutions for ionized disks with anomalous Ohmic resistivity that are completely analogous to their low-ionization counterparts. One can relate the magnetized disk model to the ' $\alpha$-viscosity' model employed in the analysis of Bell $\&$ Lin (1994) by making the identification $\alpha=\epsilon\left(V_{\mathrm{K}} / C_{\mathbf{B}}\right)$. Königl (1995) made a preliminary determination that for $\alpha \approx 10^{-3}$ (the characteristic value inferred by Bell \& Lin 1994 for FU Orionis systems in outburst) and $V_{\mathrm{K}} / C_{\mathrm{s}} \gtrsim 10$, the wind-driving disk model could be consistent with the formulation of the thermal ionization instability. A more detailed study is currently in progress, and the results may be applicable also to AGNs. ${ }^{2}$

\section{Origin of Disk Magnetic Fields}

A necessary ingredient of the MHD wind-driving disk scenario is the presence of ordered, open magnetic field lines in the disk. In the context of YSOs, some workers have explored the possibility that a dynamo-generated stellar magnetic field can couple to the disk material and drive the observed outflows (e.g., Shu et al. 1994). Although this question is still not settled, various lines of evidence point to outflows that either start far from the stellar surface (e.g., Königl \& Ruden 1993) or else occur during FU Orionis outbursts when the stellar magnetic field is evidently compressed by a strong inflow (e.g., Calvet et al. 1993). It is also clear that a magnetic field anchored at the center could not account for a global angular momentum transport in the disk. Furthermore, this concept would not even be relevant if the central object were a black hole, as is the case in AGNs. The most likely alternative origin of $\mathbf{B}$ is the interstellar field that has been advected inward either during the initial formation phase of the disk (which, in the protostellar case, was probably associated with the gravitational collapse of the parent molecular cloud core) or subsequently by accretion through the disk. ${ }^{3}$

The issue of the field distribution established during the collapse of a molecular cloud core is part of the larger question of the evolution of magnetically supported molecular clouds towards the formation of protostars and circumstellar disks. Much progress has been made in the investigation of this complex problem over the last few years (e.g., Ciolek \& Mouschovias 1995; Li \& Shu 1996), but the field configuration in a newly formed, rotationally supported, circumstellar disk still remains to be derived. There is, however, a growing recognition that a substantial fraction of the mass in many YSOs is accumulated through disk accretion well after the formation of the protostellar core, so it is conceivable that the magnetic flux distribution in the disk could be significantly modified during that phase. Flux transport through a disk has recently been considered by Lubow et al. (1994) and by Reyes-Ruiz \& Stepinski (1996), who modeled

\footnotetext{
${ }^{2}$ Note that the magnetic recoupling idea may be relevant to such outbursts even if the wind is not the dominant angular momentum transport mechanism in the disk so long as the viscosity has a magnetic origin; cf. Stone et al. 1996 and Gammie 1996.

${ }^{3}$ The possibility that the field was generated by a disk dynamo is less attractive in view of the fact that the resulting magnetic configuration would be expected to have a quadrupole-like symmetry with closed field lines (e.g., Reyes-Ruiz \& Stepinski 1996).
} 
the evolution of magnetic field lines that are anchored at large distances and advected by the gas in a viscous accretion disk. They pointed out that if the viscosity and magnetic diffusivity are based on the same physical process (e.g., turbulence), then the transport of flux will necessarily be inefficient, in that the large viscosity required for a significant inflow velocity will be associated with a large diffusivity that will inhibit advection. Lovelace et al. (1994) incorporated angular momentum transport by CDWs and showed that this can lead to efficient field advection. However, they did not take into account the feedback effect of the evolving magnetic field distribution on the accretion process and obtained possibly unrealistic runaway solutions.

Contopoulos \& Königl (1996) have attempted to solve the flux advection problem self-consistently by assuming that the angular momentum transport in the disk is controlled by the large-scale magnetic stresses and taking into account the influence of the field on the vertical structure of the disk. Since the angular momentum can in principle be removed through torsional Alfvén waves driven into the ambient medium, they do not require that the CDW launching condition be satisfied at the disk surfaces, although they take into account the contribution of the outflow term in the mass conservation equation when this condition is satisfied. They consider a resistive, locally isothermal, Keplerian disk that is initially threaded by a uniform vertical magnetic field anchored at a large distance from the disk, and follow the evolution of the disk under the electromotive force induced by this field (see Fig. 1). They prescribe the radial distribution of the magnetic diffusivity, the temperature, and the radial electric current in the disk, and adopt a value for the resistive load of the external medium through which the electric circuit closes. The solution of the induction equation is simplified by treating the disk at each radius as a uniform slab, but the detailed vertical structure is taken into account in calculating the mass outflow rate through the sonic point. Work is currently in progress to incorporate a realistic prescription of the magnetic diffusivity into this model and use the results to investigate magnetic flux transport in protostellar disks.

\section{Observational Implications in YSOs and AGNs}

Disk-driven centrifugal winds have several unique characteristics that may have important implications to the observational properties of YSOs and AGNs. In particular, such outflows are distinguished by a highly stratified density and velocity structure, a high momentum discharge that results in the efficient uplifting of dust from the associated disks, and, in the case of low-luminosity YSOs, a robust ambipolar diffusion-heating mechanism that raises the temperature in the inner regions of the wind (on scales $\gtrsim 10^{2} \mathrm{AU}$ ) to $\gtrsim 10^{4} \mathrm{~K} .{ }^{4}$ The strong $\rho$ and $V$ stratification is a consequence of the rapid acceleration of the wind near the disk surface. The uplifted dust is evaporated by the central radiation field within the sublimation radius $r_{\text {sub }}\left(\sim 0.1\left[L / L_{\odot}\right]^{0.5}\right.$ AU for a YSO and $\sim 0.1\left[L / 10^{44} \text { ergs }^{-1}\right]^{0.5}$ pc for an AGN, where $L$ is the bolometric luminos-

\footnotetext{
${ }^{4}$ This mechanism could in principle operate also in the self-shielding regions of higher-luminosity objects that drive high- $\dot{M}_{\text {wind }}$ outflows from their disk surfaces.
} 

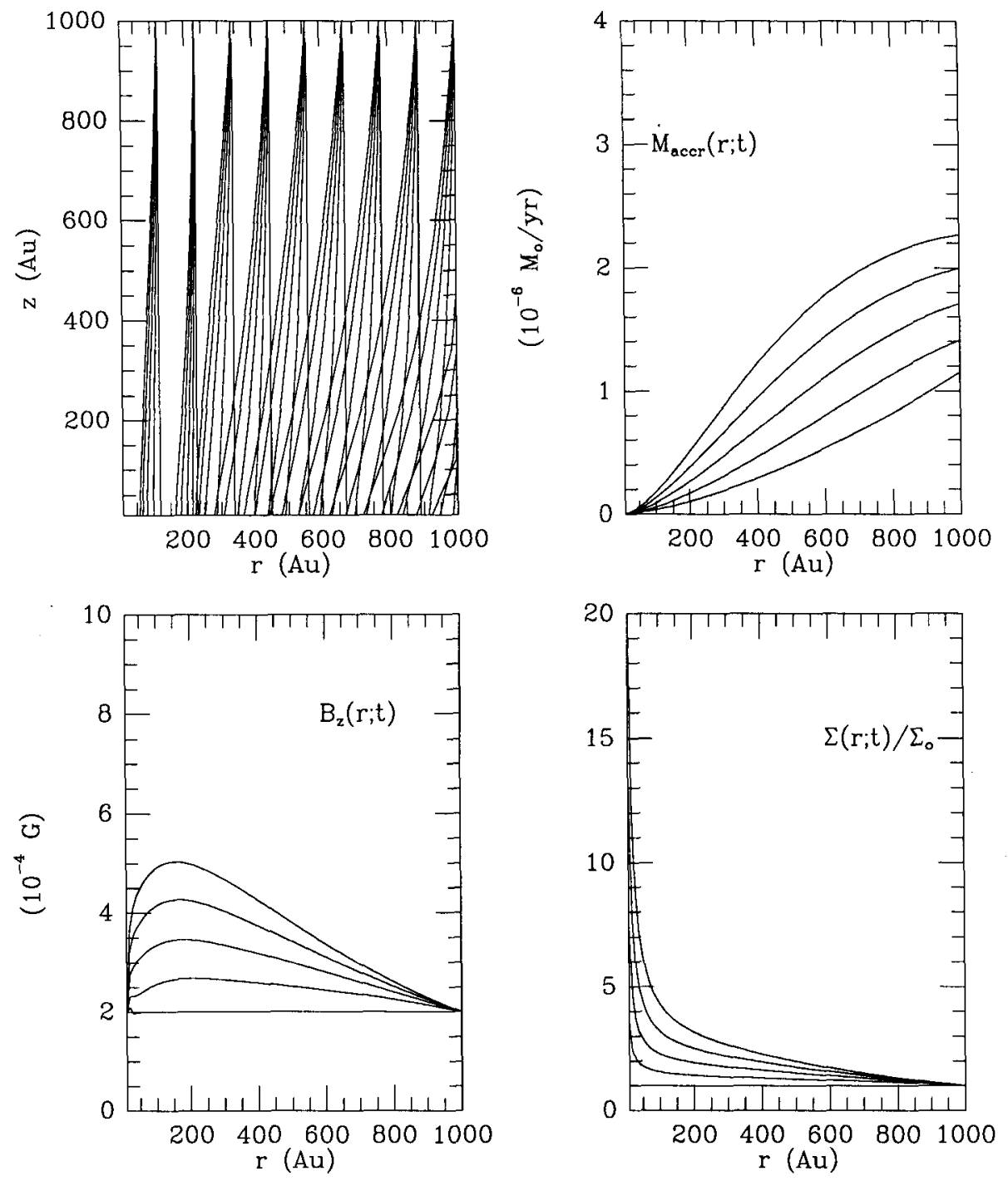

Figure 1. An illustrative example of the evolution of a Keplerian accretion disk around a solar-mass YSO that is surrounded by a forcefree magnetosphere. The disk extends between 10 and $10^{3} \mathrm{AU}$ and initially has a uniform surface density $\Sigma_{0}=7 \times 10^{-3} \mathrm{~g} \mathrm{~cm}^{-2}$ and is threaded by a uniform $0.2 \mathrm{mG}$ vertical magnetic field that is anchored at a large distance above its surface. The 4 panels show, respectively, the poloidal field line shape, the mass accretion rate, the axial field strength, and the disk surface density (calculated without taking into account the effect of mass outflow), plotted at $10^{3}$-yr intervals. As the disk accretes, extra flux is allowed to be transported in from larger radii. The electric current outside the disk is assumed to flow along the axis and the outer boundaries, so $B_{\phi} \propto r^{-1}$ everywhere. 
ity), but at larger distances the dust survives and exhibits the same density distribution as the gas in which it is embedded. ${ }^{5}$

The stratified dust distribution predicted by this model leads to angledependent obscuration that may account for the obscuring 'tori' that have been invoked in the Seyfert 1/Seyfert 2 unification scheme as well as in the analogous schemes proposed for relating other types of AGNs (Königl \& Kartje 1994). Furthermore, such a dust distribution accounts naturally for the optical/UV polarization properties of AGNs (Kartje 1995) and is also consistent with the centrosymmetric + parallel equatorial polarization pattern that characterizes many YSOs. In addition, the scattering and reprocessing of the central continuum radiation by the dust in the wind and the disk could give rise to the observed infrared spectra in both YSOs and AGNs (e.g., Königl \& Kartje 1994).

The magnetic field that accelerates the mostly neutral gas in CDWs from weakly ionized disks is coupled to the bulk of the matter through ion-neutral collisions. As was demonstrated by Safier (1993a), the accompanying collisional dissipation could be the dominant heating mechanism in winds from solar-mass YSOs, and, for flow lines that originate within $\lesssim 3 \mathrm{AU}$ from the star, it could lead to temperatures and electron densities that are consistent with the values inferred from the forbidden line emission in T Tauri stars on scales of $\lesssim 10^{2} \mathrm{AU}$. Disk-driven winds could thus contribute to the observed forbidden line emission, especially to its low-velocity component (see Safier 1993b). This mechanism may also play an important role in producing several other emission signatures of YSOs, most notably the thermal radio radiation (Martin 1996; see Fig. 2).

Acknowledgments. I am grateful to John Contopoulos, John Kartje, Steve Martin, Pedro Safier, and Mark Wardle for their valuable input to the work described in this contribution. This research was supported in part by NASA grant NAG5-2766.

\section{References}

Bell, K. R., \& Lin, D. N. C. 1994, ApJ, 427, 987

Blandford, R. D. 1993, in Astrophysical Jets, ed. M. Livio, C. O’Dea, \& D. Burgarella (Cambridge: Cambridge Univ. Press), 15

Blandford, R. D., \& Payne, D. G. 1982, MNRAS, 199, 883

Cao, X., \& Spruit, H. C. 1994, A \& A, 287, 80

Calvet, N., Hartmann, L., \& Kenyon, S. J. 1993, ApJ, 402, 623

Ciolek, G. E., \& Mouschovias, T. Ch. 1995, ApJ, 454, 194

Contopoulos, J. 1995, ApJ, 450, 616

Contopoulos, J., \& Königl, A. 1996, in preparation

Ferreira, J., \& Pelletier, G. 1995, A \& A, 295, 807

Gammie, C. F. 1996, ApJ, 457, 355

\footnotetext{
${ }^{5}$ The high-momentum-discharge continuous outflows could also play a key role in uplifting and accelerating discrete, dense clouds from the vicinity of the disk surface. Some observational consequences of this possibility are discussed in the contributions of Kartje \& Königl and Kartje, Königl, \& Elitzur to these Proceedings.
} 

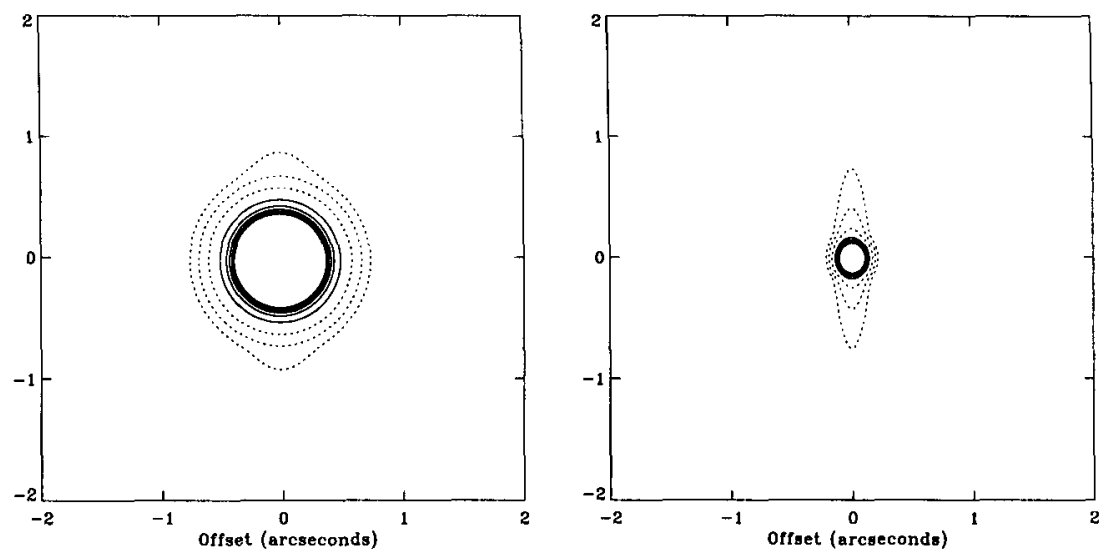

Figure 2. Synthetic $5 \mathrm{GHz}$ radio maps for a representative CDW model corresponding to a $\mathrm{T}$ Tauri star at a distance of $160 \mathrm{pc}$. The inclination of the symmetry axis with respect to the line of sight and the FWHM of the convolved Gaussian beam are $\left\{45^{\circ}, 0.35^{\prime \prime}\right\}$ and $\left\{85^{\circ}\right.$, $\left.0.1^{\prime \prime}\right\}$ for the left and right panels, respectively (from Martin 1996).

Kartje, J. F. 1995, ApJ, 452, 565

Königl, A. 1994, in Theory of Accretion Disks - 2, ed. W. J. Duschl et al. (Dordrecht: Kluwer), 53 . 1995, RMxAA (Conf. Ser.), 1, 275

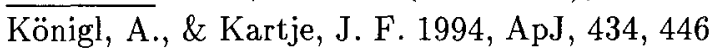

Königl, A., \& Ruden, S. P. 1993, in Protostars and Planets III, ed. E. H. Levy \& J. I. Lunine (Tucson: University of Arizona Press), 641

Königl, A., \& Wardle, M. 1996, MNRAS, 279, L61

Li, Z.-Y. 1995, ApJ, 444, 848

$$
\text { . 1996, ApJ, 465, } 855
$$

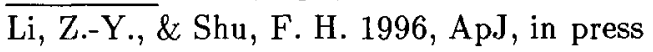

Lovelace, R. V. E., Berk, H. L., \& Contopoulos, J. 1991, ApJ, 379, 696

Lovelace, R. V. E., Romanova, M. M., \& Newman, W. I. 1994, ApJ, 437, 136

Lubow, S. H., Papaloizou, J. C. B., \& Pringle, J. 1994, MNRAS, 267, 235

Martin, S. C. 1996, ApJ, in press

Reyes-Ruiz, M., \& Stepinski, T. F. 1996, ApJ, 459, 653

Safier, P. N. 1993a, ApJ, 408, 115

$$
\text { . 1993b, ApJ, 408, } 148
$$

Shu, F. H., Najita, J., Ostriker, E., Wilkin, F., Ruden, S., \& Lizano, S. 1994, ApJ, 429, 781

Stone, J. M., Hawley, J. F., Gammie, C. F., \& Balbus, S. A. 1996, ApJ, 463, 656

Wardle, M., \& Königl, A. 1993, ApJ, 410, 218 